\title{
Superconducting properties of a mesoscopic parallelepiped with anisotropic surface conditions
}

\author{
J. Barba-Ortega ${ }^{\mathrm{a}, *}$, Edson Sardella ${ }^{\mathrm{b}, \mathrm{c}}$ \\ a Departamento de Física, Universidad Nacional de Colombia, Bogotá, Colombia \\ b UNESP-Universidade Estadual Paulista, Departamento de Física, Caixa Postal 473, Bauru-SP, Brazil \\ c UNESP-Universidade Estadual Paulista, IPMet-Instituto de Pesquisas Meteorológicas, CEP 17048-699 Bauru, SP, Brazil
}

\section{A R T I C L E I N F O}

\section{Article history:}

Received 19 May 2015

Received in revised form 9 October 2015

Accepted 10 October 2015

Available online 20 October 2015

Communicated by L. Ghivelder

\section{Keywords:}

Ginzburg-Landau

De Gennes parameter

Mesoscopic

Anisotropic surfaces

\begin{abstract}
A B S T R A C T
We consider a mesoscopic superconducting parallelepiped with different boundary conditions on different parts of the surface, $x y, x z$ and $y z$ surface planes. This is realized by considering different values of the de Gennes extrapolation length $b$ on different surfaces of the sample. Our investigation was carried out by solving the three-dimensional (3D) time dependent Ginzburg-Landau (TDGL) equations. We studied the local magnetic field, order parameter, and both the magnetization and vorticity curves as functions of the external applied magnetic field for different values of $b$ on the surfaces of the sample. We show that this surface anisotropy has very strong influence on the vortex configurations and the magnetization as a function of the external applied magnetic field, both experimentally accessible.
\end{abstract}

(c) 2015 Elsevier B.V. All rights reserved.

\section{Introduction}

A very important consequence to consider a 3D superconductor sample in a homogeneous external applied magnetic field is the demagnetization effect. The study of such systems by using the 3D TDGL equations requires an enormous computational effort. However, from the quantitative point of view it is much more advantageous than the two-dimensional (2D) TDGL model, since we obtain precisely the critical fields, the vortex configurations, energy and magnetization stability curves, etc. There are many experimental (see for instance [1-6]) and theoretical (see for instance [7-10]) studies for 3D systems. In all these theoretical studies, the Ginzburg-Landau model has been proven to give a good account of the superconducting properties in samples of several geometries, i.e., disks with finite thickness and spheres [11,12], shells [13], cone [14], thin circular sectors, thin disks and SQUID geometry [15-18].

As is well-known from the microscopic theory, the GinzburgLandau approach gives accurate results close to the critical temperature $T_{c}$. However, from the experience it is well known that it is also capable of gives reasonable results beyond this limit.

In a recent work, the authors of this paper made a comparative study between the 2D and 3D Ginzburg-Landau models. We de-

\footnotetext{
* Corresponding author.

E-mail address: josejbarba@gmail.com (J. Barba-Ortega).
}

termined an analytical dependence of the thermodynamic fields and the magnetization as functions of the lateral dimension of the superconductor [19]. There, we have considered only the more common situation of a superconductor-vacuum interface.

In this paper, we have gone further by investigating the effects of the boundary conditions of the superconducting order parameter at the surface of the specimen; they modify the structural and magnetic properties of the vortex state as the size of the sample is comparable to the coherence length $\xi$ or the London penetration depth $\lambda$ [20]. We consider a superconductor covered by a very thin layer of a different material. Three types of interfaces are taken into account: superconductor-vacuum, superconductornormal metal, superconductor-superconductor at a higher critical temperature. Either we employ the same boundary conditions in all the six faces of a parallelepiped geometry (isotropic interface type), or we combine them in several different manners (anisotropic interface type).

The paper is outlined as follows. In Section 2 we briefly describe the theoretical formalism used to study a mesoscopic superconducting parallelepiped in the presence of an external applied magnetic field. We also present all the seven scenarios of boundary conditions which will be undertaken. Then, in Section 4 we present the results coming out from the numerical solution of the TDGL equations for the magnetization, vorticity, vortex configurations and local magnetic field profiles. 


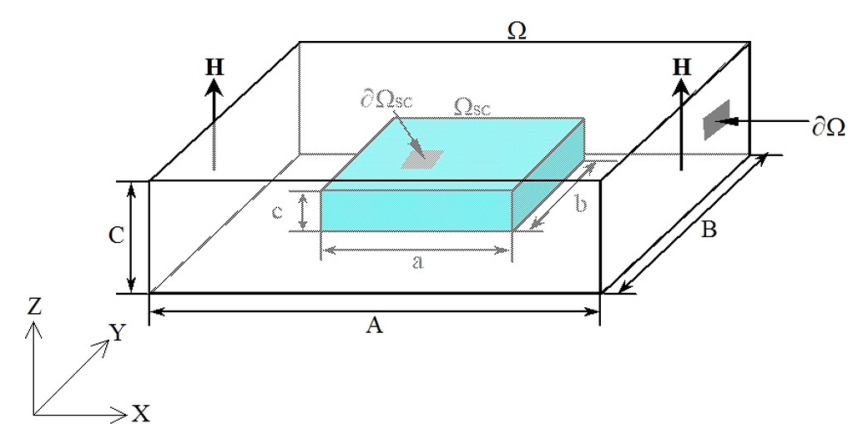

Fig. 1. (Color online.) Schematic view of the geometry of the system under investigation. The meaning of all symbols are described in the text.

\section{Theoretical formalism}

The geometry of the problem that we investigate is illustrated in Fig. 1. The domain $\Omega_{s c}$ is filled by the mesoscopic superconducting parallelepiped of thickness $c$ and lateral sizes $a$ and $b$. The interface between this region and the vacuum is denoted by $\partial \Omega_{s c}$. Because of the demagnetization effects, we need to consider a larger domain $\Omega$ of dimensions $A \times B \times C$, such that $\Omega_{s c} \subset \Omega$. The vacuum-vacuum interface is indicated by $\partial \Omega$. We consider a mesoscopic superconducting parallelepiped in the presence of an uniform applied magnetic field $\mathbf{H}$ along the $z$ direction. The domain $\Omega$ is taken sufficiently large such that the local magnetic field equals the applied field $\mathbf{H}$ at the surface $\partial \Omega$ (see Ref. [19] for more details). The general form of the time dependent Ginzburg-Landau equations in dimensionless units are given by:

$$
\begin{aligned}
& \frac{\partial \psi}{\partial t}=-(-i \nabla-\mathbf{A})^{2} \psi+\psi\left(1-|\psi|^{2}\right), \text { in } \Omega_{s c}, \\
& \frac{\partial \mathbf{A}}{\partial t}=\left\{\begin{array}{l}
\mathbf{J}_{s}-\kappa^{2} \nabla \times \nabla \times \mathbf{A}, \quad \text { in } \Omega_{s c}, \\
-\kappa^{2} \nabla \times \nabla \times \mathbf{A}, \quad \text { in } \Omega \backslash \Omega_{s c},
\end{array}\right.
\end{aligned}
$$

where

$$
\mathbf{J}_{s}=\operatorname{Re}[\bar{\psi}(-i \nabla-\mathbf{A}) \psi]
$$

is the superconducting current density.

In Eqs. (1)-(3) dimensionless units were introduced as follows: the order parameter $\psi$ is in units of $\psi_{\infty}=\sqrt{-\alpha / \beta}$, the order parameter at the Meissner state, where $\alpha$ and $\beta$ are two phenomenological constants; length is in units of the coherence length $\xi$; time is in units of the Ginzburg-Landau characteristic time $t_{G L}=\pi \hbar / 8 K_{B} T_{c}$; magnetic field is in units of $H_{c 2}$, the bulk upper critical field; the vector potential $\mathbf{A}$ is in units of $\xi H_{c 2}$; $\kappa=\lambda / \xi$ is the Ginzburg-Landau parameter.

The phase diagram of mesoscopic superconductor is strongly influenced by the boundary conditions for the order parameter. In general, they are given by the de Gennes boundary conditions:

$$
\begin{aligned}
\mathbf{n} \cdot(i \nabla+\mathbf{A}) \psi & =-\frac{i}{b} \psi, \quad \text { at } \partial \Omega_{s c}, \\
\nabla \times \mathbf{A} & =\mathbf{H}, \quad \text { at } \partial \Omega,
\end{aligned}
$$

where $\mathbf{n}$ is the unit vector outward normal to the superconductormedium interface, $b$ is the de Gennes surface extrapolation length which describes this medium; as we have stated previously, $\Omega$ is a domain sufficiently large such that the local magnetic field $\mathbf{h}=$ $\boldsymbol{\nabla} \times \mathbf{A}$ equals the external applied magnetic field $\mathbf{H}$.

It must be emphasized that the space between the interfaces $\partial \Omega_{s c}$ and $\partial \Omega$ is not filled by any material. The superconductor is covered by a very thin layer of another material which is contained in the domain $\Omega_{s c}$. This layer is described by the de Gennes extrapolation length $b$.

In order to solve equations (1)-(3) numerically, we used the link-variable method as sketched in Refs. [21,22]. This is not the unique method available to solve these equations. A method valid for very thin films has been devised in Ref. [23], in which fast Fourier transform combined with the link-variables was employed. Although we used the TDGL equations, we are concerned only with the stationary state; they are used only as a relaxation method to obtain the equilibrium state.

By varying the values of $b$, we can change the nucleation field and the critical current. From the microscopic point of view, it is possible to show that $b$ depends on the properties of the interface; it is maximum for an ideal surface with the mirror reflection of quasi-particles and minimum for the rough surface with the diffusive reflection [24-27]. The values of $b$ can be estimated according to Refs. $[28,29]$. The superconductor-vacuum interface, $b \rightarrow \infty$, has been extensively studied by many authors (see for instance [30-35]). The case in which $b>0$ is used, the order parameter is suppressed in the vicinity of the sample surface, a normal metal. For the superconductor-normal metal interface $b$ is always small, $b \sim \xi$, because of diffusion of normal electrons from the metal to the superconductor. The enhancement of the order parameter is reached at the interface by using negative values of $b$. This can be realized by covering the superconductor sample with a very thin layer of another superconductor having a higher critical temperature [36]. This can also be achieved by covering it with a semiconductor, such that there is an overlap of the band gap of the semiconductor with the superconducting gap. In this paper, we will study a mesoscopic superconducting parallelepiped for several types of interfaces.

According to Fig. 2, we have considered the three following scenarios:

- Case 1: the upper and lower faces of the sample, $x y$ planes, are in contact with a metallic material, $b=5 \xi$; or a superconductor material at higher critical temperature, $b=-5 \xi$; the $x z$ and $y z$ planes are in contact with vacuum, $b \rightarrow \infty$; left panels of the figure.

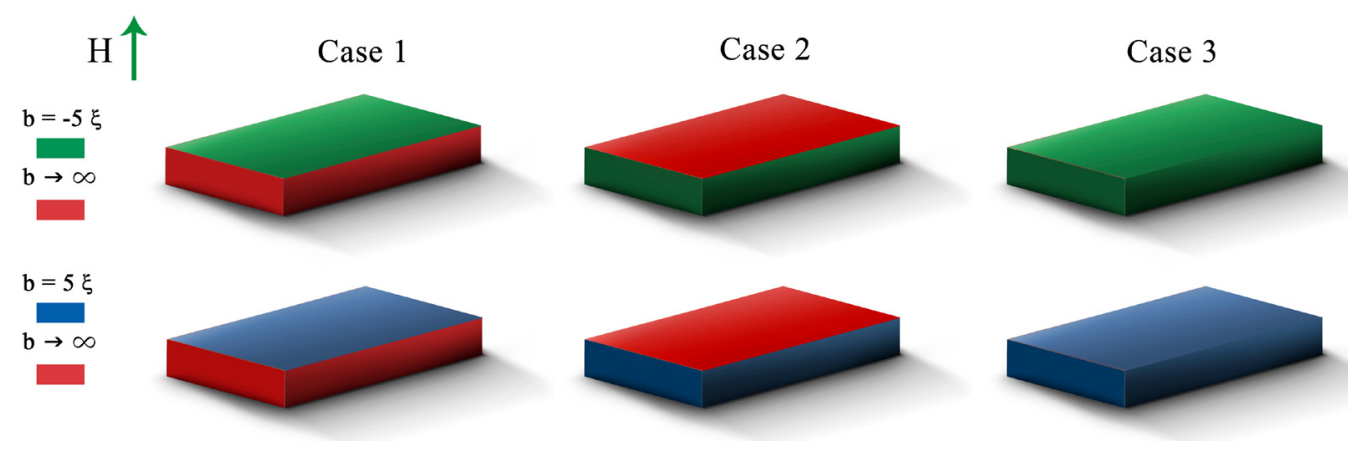

Fig. 2. Layout of the studied samples; the external applied magnetic field $\mathbf{H}$ is pointed perpendicular to the $x y$ plane. 

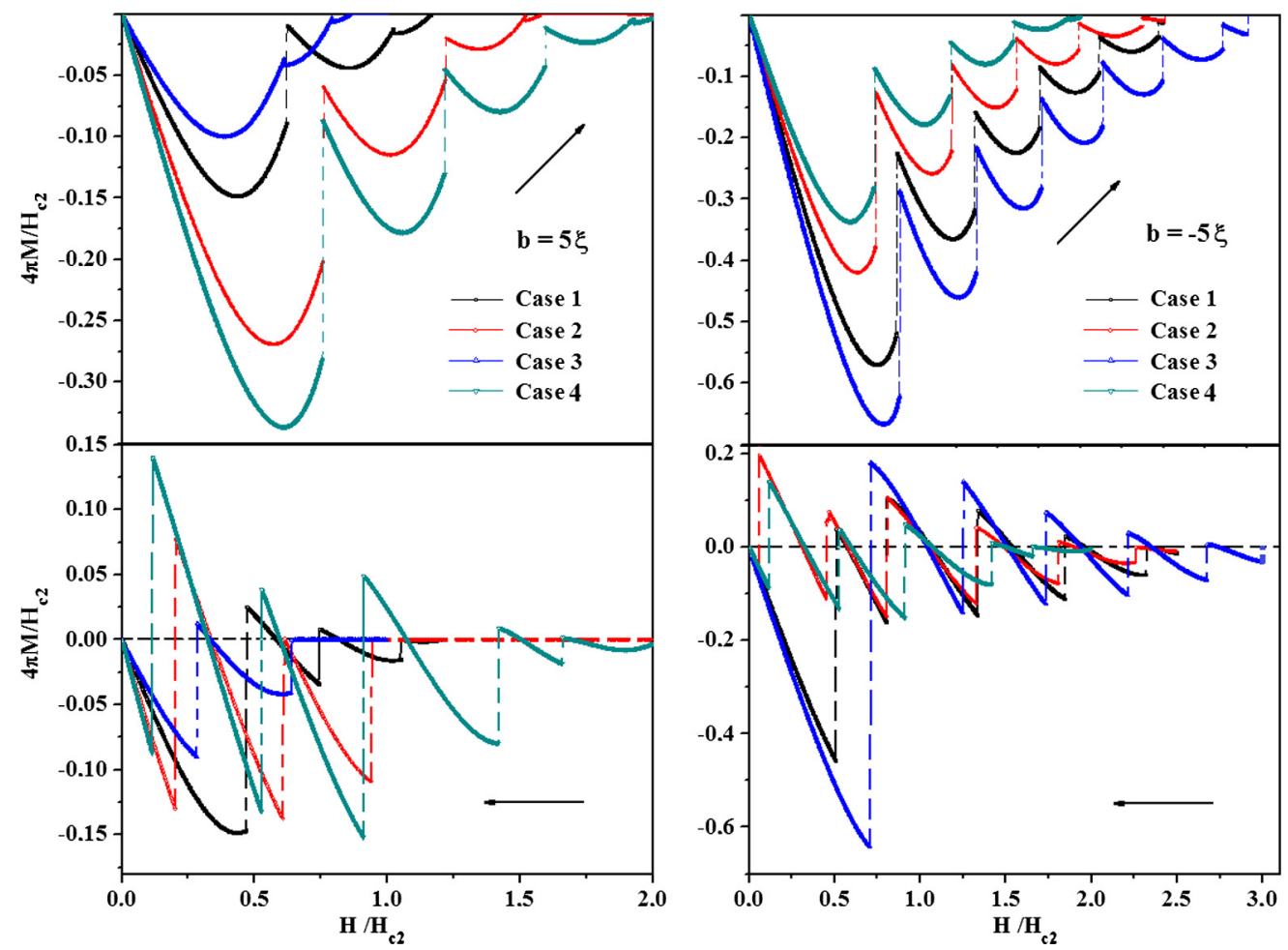

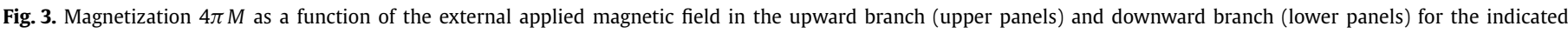
values of $b$.

- Case 2: the $x y$ planes are in contact with vacuum, $b \rightarrow \infty$; the $x z$ and $y z$ planes are either in contact with a metallic material, $b=5 \xi$, or a superconductor at higher critical temperature material, $b=-5 \xi$; middle panels of the figure.

- Case 3: all planes are either in contact with a metallic material, $b=5 \xi$, or a superconductor at higher critical temperature material, $b=-5 \xi$; right panels of the figure.

- Case 4: all planes are in contact with vacuum $b \rightarrow \infty$; not shown in Fig. 2.

Case 4 is used for comparison purposes. Thus, case 4 and both cases 3 are isotropic interface type. All the other ones are anisotropic interface type.

In Ref. [19] we described in details the differences between the 2D and 3D Ginzburg-Landau models. In the 2D TDGL equations, all physical quantities are taken invariant along the $z$ direction, that is, only $x z$ and $y z$ faces are taken into account. As a consequence, there should be no difference: (a) between $b=5 \xi$ and $b=-5 \xi$, case 1 , (b) between cases 2 and 3 for the respective values of $b= \pm 5 \xi$. Thus, only upon using the 3D TDGL equations we could detect the influence of distributing the boundary conditions non-uniformly throughout all the six faces of the parallelepiped.

In the next Section, we present a detailed comparative study of the intra cases (negative and positive values of $b$, columns of Fig. 3), and inter cases (fixed values of $b$, rows of Fig. 3).

\section{Results and discussion}

In order to solve the 3D TDGL equations, the size of the simulation box $\Omega$ was taken as $19 \xi \times 19 \xi \times 11 \xi$ and the superconductor inside has the size $6 \xi \times 6 \xi \times 1 \xi$; we used $\kappa=1$ and a mesh with grid size $\Delta x=\Delta y=\Delta z=0.1 \xi$. This choice of the dimension of the simulation box and the superconducting parallelepiped assures that the surfaces $\partial \Omega_{s c}$ and $\partial \Omega$ are sufficiently far from one each other. This guarantees that the local field will be uniform faraway form the superconducting domain. The values of the de Gennes parameter are quoted in Fig. 2.

Figs. 3 and 4 show the magnetization $4 \pi M$ and vorticity $N$, respectively, as functions of the external applied magnetic field when $H$ is increasing (upper panels) and decreasing (lower panels) for the four considered cases stated previously. As can be seen from these figures, for $b=5 \xi$, the lower critical fields are $H_{1}=0.622 H_{c 2}, H_{1}=0.760 H_{c 2}, H_{1}=0.614 H_{c 2}, H_{1}=0.7534 H_{c 2}$ for cases $1,2,3$, and 4 respectively. Thus, the lower critical field is approximately equal for cases 2 and 4 , and cases 1 and 3. These similarities are not difficult to understand if we notice that the thickness of the sample is rather small. Therefore, the lateral interfaces ( $x z$ and $y z$ planes) do not have a significant influence on shielding the superconductor from the first nucleation of vortices; what really matters are the upper and lower interfaces. ${ }^{1}$ This is a very important advantage of the 3D TDGL model, since with the 2D counterpart we would not be able to keep these similarities because in both comparisons the lateral interfaces are different. The upper critical field strongly depends on the boundary conditions. From the left upper panel of Fig. 3 we obtain $\mathrm{H}_{2}=1.602 \mathrm{H}_{c 2}, 1.198 \mathrm{H}_{c 2}, 0.880 \mathrm{H}_{c 2}$ for cases 2,1 and 3 respectively. Then, we can conclude that, the larger the area of the superconductor is covered by a metal, the smaller the value of $\mathrm{H}_{2}$ is. Again, we see the importance of the 3D Ginzburg-Landau model, since from the $2 \mathrm{D}$ perspective there should be no difference between cases 2 and 3.

Still concerning Fig. 3, we see that for the $b=-5 \xi$ curves, the lower critical field is the same for cases 2 and $4, H_{1}=0.756 H_{c 2}$; while for cases 1 and 3 this critical field are approximately the same, $H_{1}=0.890 H_{c 2}$ and $H_{1}=0.911 H_{c 2}$ respectively. Then, sim-

\footnotetext{
1 This result is valid only for a very thin superconductor. In this case, the upper and lower surfaces are so close to one each other that they have a strong influence inside the superconductor, inclusive in the regions near the lateral surface. Although we have not considered very thick samples, we expect that in this limit the screening properties would be the opposite from what we have found.
} 

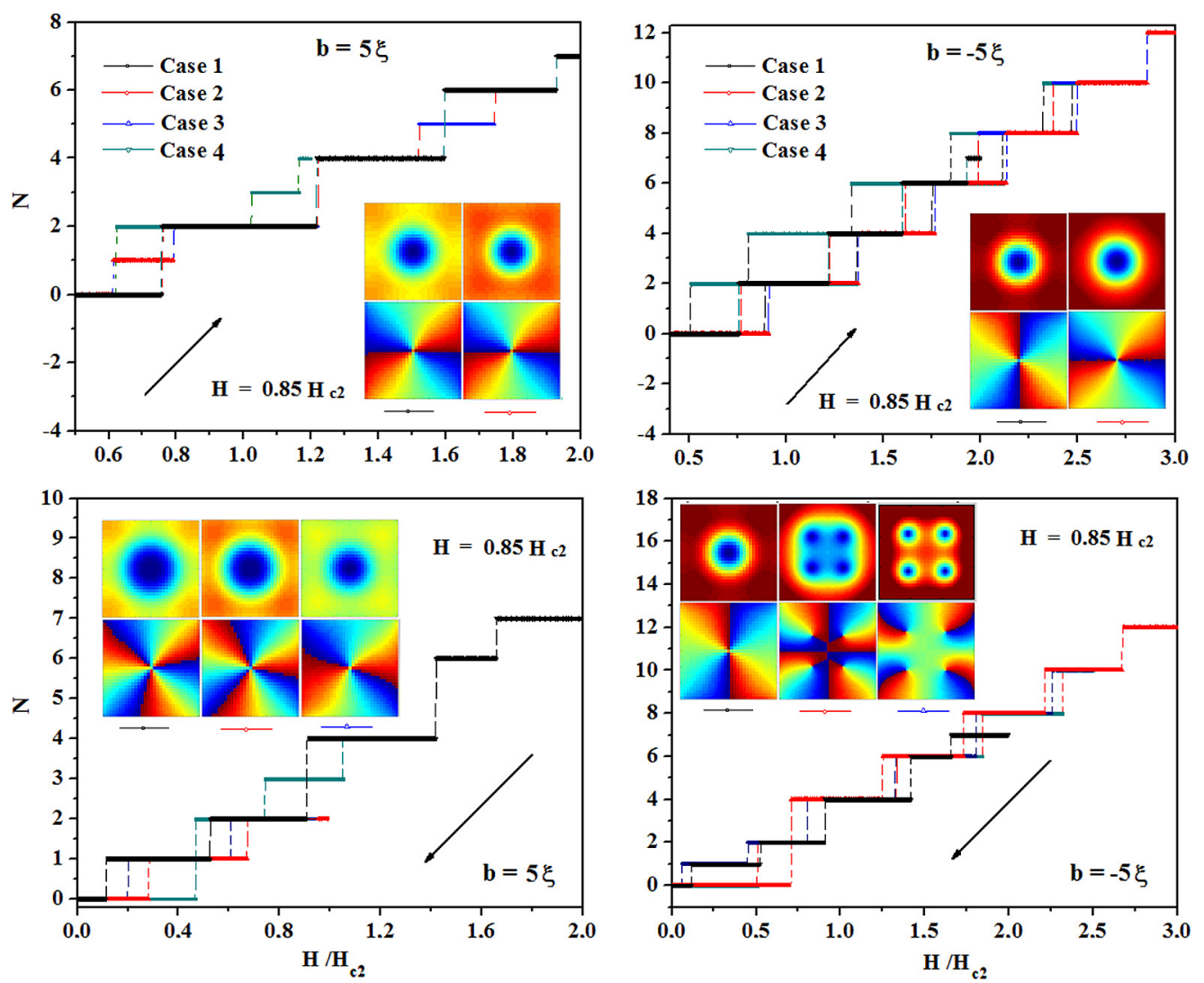

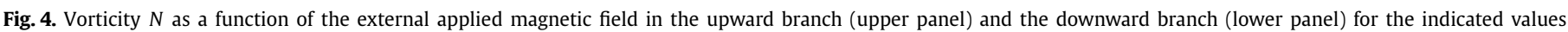

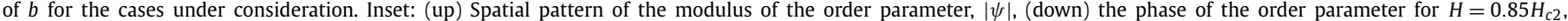
on plane $z=C / 2$. (For interpretation of the colors in this figure, the reader is referred to the web version of this article.)

ilarly to the $b=5 \xi$ situation, the lateral interfaces do not play a significant role on the values of the lower critical fields. For the upper critical field we find $\mathrm{H}_{2}=3.150 \mathrm{H}_{c 2}, 2.608 \mathrm{H}_{c 2}, 2.348 \mathrm{H}_{c 2}$, for the cases 3, 2 and 1, respectively. In opposition to the situation of $b=5 \xi$, it seems that there is not a direct relationship between the value of $\mathrm{H}_{2}$ and the area of the superconductor covered by another superconductor at higher temperature, since the upper critical field is larger for case 2 than for case 1, although the difference is small. Again, we notice that the 2D TDGL model could not detect the differences between cases 2 and 3 .

Let us now analyze the downward branch of the magnetization curves (see lower panels of Fig. 3). As we have observed above, cases 1 and 3 present a less efficient barrier for first penetration of vortices than cases 2 and 4 for $b=5 \xi$. On the other hand, for $H$ decreasing, the systems of cases 1 and 3 expel all the vortices at larger external applied magnetic fields (see lower left panel). Then, it seems that those samples most covered by a metal exert more attraction to the vortices inside when $H$ is decreasing. Concerning the $b=-5 \xi$ situation (lower right panel), we see that a similar scenario occurs.

Next, we present some transitions in the upward and downward branches of the vorticity as a function of the external applied magnetic field $H$ (see Fig. 4). The arrows indicate when $H$ is increasing (upper panels) and decreasing (lower panels). The vortex position can be found from the singularity of phase of the order parameter, located at the interior of the sample where it presents a change of $2 \pi$ in a closed path (the color changes from blue to red in the anticlockwise sense). As we have strengthened above, the full vortex expulsion fields are different in the downward branch of the magnetic field for each considered case. We can see this effect more clearly when $N$ vanishes in the downward branch. Let us denote this magnetic field by $H_{p}$. Just to quote, we have the following scenarios for the final vortex transitions when $H$ is decreasing for $b=5 \xi: N=2 \rightarrow 0, H_{p}=0.470 H_{c 2}$, case 1 ; $N=1 \rightarrow 0, H_{p}=0.202 H_{c 2}$, case $2 ; N=1 \rightarrow 0, H_{p}=0.284 H_{c 2}$, case 3. Now, when the boundary condition $b=-5 \xi$ is employed: $N=2 \rightarrow 0, H_{p}=0.508 H_{c 2}$, case $1 ; N=1 \rightarrow 0, H_{p}=0.060 H_{c 2}$, case $2 ; N=4 \rightarrow 0, H_{p}=0.710 H_{c 2}$, case 3 .

Now we show some snapshots of the modulus of order parameter and its corresponding phase in the $z=0$ plane (see insets of Fig. 4). In order to make a comparison between all cases on equal foot, we chose the same value of $H$. For $H=0.85 H_{c 2}$ we see that the vorticity $N=2$ is the same for both $b=5 \xi$ and for $b=-5 \xi$, cases 1 and 2 . In addition, the vortex configurations are identical, an $N=2$ giant vortex at the center, although their size are quite distinct. On the other hand, for the same value of $H$ when the external applied magnetic field is decreasing, we have different situations for both values of $b$, and also between all cases 1 , 2 , and 3 for the same value of $b$. For $b=5 \xi$ we have: $N=3$ giant vortex state, case $1 ; N=3$ giant vortex state, case 2 ; and $N=2$ giant vortex state, case 3 . As for $b=-5 \xi$ we have: $N=2$ giant vortex state, case $1 ; N=4$ fourfold symmetry vortex state, case 2 ; and $N=4$ fourfold symmetry vortex state, case 3 . For a fixed value of $b$, even when the vorticity is the same, the dimensions of the vortices are visibly different. Thus, we see that the boundary conditions have a strong influence on the vortex configurations of a superconductor and they can be detected only with the use of the 3D TDGL equations. Until some time ago, the existence of giant vortex was a controversial issue, although all the numerical solutions of the TDGL equations pointed out that confinement effects do lead to this novel state (see for instance Refs. $[31,37]$ and references therein). Experiment on $\mathrm{Pb}$ films by using scanning Hall probe microscopy were not conclusive with respect to the giant vortex state (see for instance Ref. [38]). However, after 


\section{Case 4}
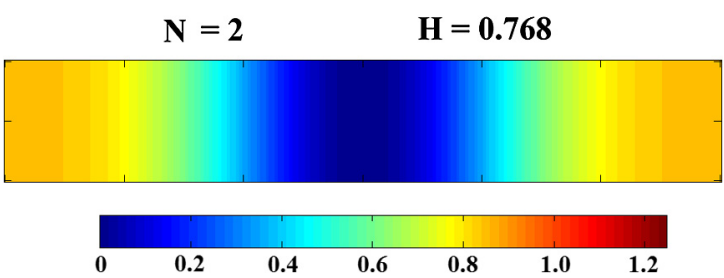

Fig. 5. Intensity of the order parameter on the $x z$ plane $(y=B / 2)$ for the isotropic case 4 . At the center we have an $N=2$ giant vortex state.

a direct scanning tunneling spectroscopy experiment on ultrathin single crystal of $\mathrm{Pb}$ conducted by Cren et al. [39], it remains rather doubt about the occurrence of ultradense arrangements of single Abrikosov vortices (giant vortices). Also, very recently, Lukyanchuk et al. [4] show some magnetization measurements accompanied by numerical simulations which reveal both fission and fusion of multi-quanta vortex droplet.

We also have investigated how the order parameter and the size of the vortex core change along the $z$ direction (on the $x z$ plane, $y=B / 2$ ) for all isotropic/anisotropic interface types under investigation. In Fig. 5 we show the modulus of $\psi$ for case
4 (isotropic surfaces, $b=\infty$ ). We see that the order parameter is uniform from the bottom to the top surface. This is rather expected since the film thickness is very small. In Fig. 6 we have plotted the remaining cases 1,2 , and 3 . It can be easily seen that we can obtain case 1 from 4 by maintaining the lateral surfaces unchanged and substituting the top and bottom $b=\infty$ surfaces to either $b=5 \xi$ or $b=-5 \xi$. In this case we have a different picture. As can be seen, now we have a significant variation of the order parameter throughout the thickness of the film. Notice that the case $b=5 \xi$ has a larger core size than its counterpart $b=-5 \xi$; and for this latter case the regions near the lateral surfaces of the superconductor remain much more preserved, even for higher values of $H$. From case 4, if we keep the top and bottom surfaces at $b=\infty$ and replace the lateral surfaces with $b= \pm 5 \xi$ we obtain case 2 ; now, it seems that the central core has nearly the same size for both negative and positive values of $b$. However, as expected, the regions near the lateral surfaces of the order parameter are more preserved for a parallelepiped with a superconductorsuperconductor at a higher critical temperature interface than for a superconductor-normal metal one. Lastly, we consider case 3 in which we have either $b=5 \xi$ or $b=-5 \xi$ isotropic surfaces. Contrary to the isotropic surfaces of case 4 , even small, now we notice some variations of the order parameter in the $z$ direction near the

\begin{tabular}{|c|c|c|c|c|c|c|}
\hline \begin{tabular}{|l|}
${ }^{2}$ \\
${ }^{\prime}$
\end{tabular} & & se 1 & & & & \\
\hline$\sqrt{x}$ & $\mathbf{N}=\mathbf{2}$ & $H=0.892$ & $\mathrm{~N}=\mathbf{2}$ & $H=0.868$ & $N=1$ & $H=0.912$ \\
\hline$b=-5 \xi$ & & & & & & \\
\hline \multirow[b]{2}{*}{$\mathbf{b}=\mathbf{5} \xi$} & $\mathbf{N}=\mathbf{2}$ & $H=0.680$ & $N=\mathbf{2}$ & $H=0.666$ & $N=\mathbf{2}$ & $H=0.680$ \\
\hline & & & & & & \\
\hline
\end{tabular}

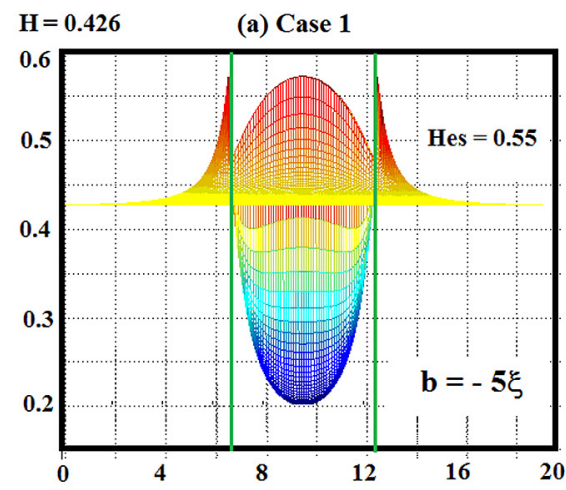

Fig. 6. The same as Fig. 5 for all cases of Fig. 2.

(b) Case 2
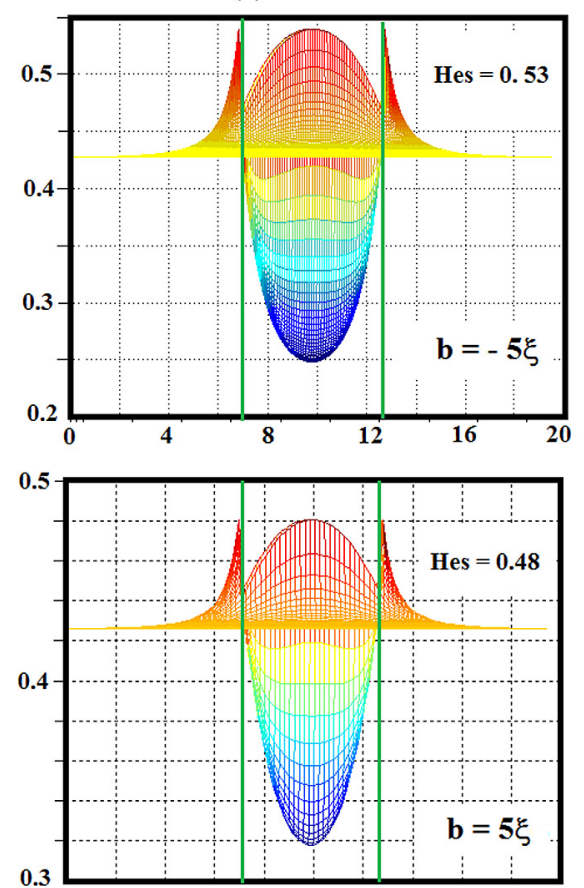

0.3 (c) Case 3
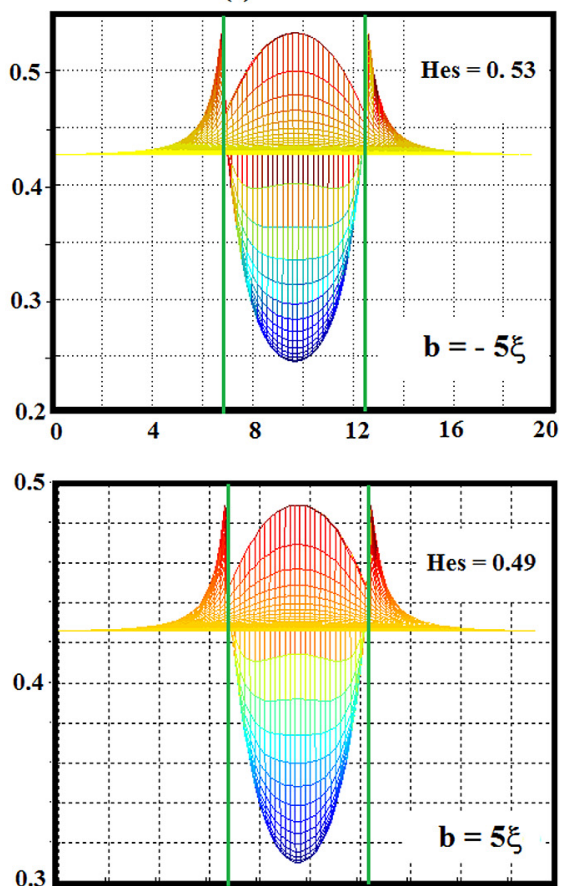

0.3

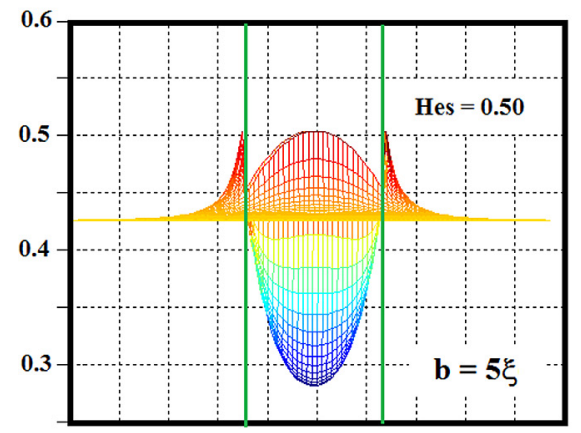

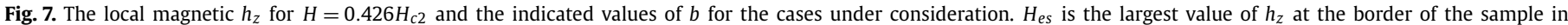
the $y=0, B$ plane. All panels are the frontal view of a 3D graphics of the local field magnetic intensity at the $z=C / 2$ plane. 
border of the vortex. The central cores seem to have the same size, but outside this region the order parameter is less destroyed for $b$ negative than positive, as was expected.

Finally, in Fig. 7 we present the local magnetic field $h_{z}$ profiles in the $z=C / 2$ plane for $H=0.426 H_{c 2}$. We see that the demagnetization effects are very enhanced at the border of the superconducting sample in the Meissner state, no matter if the boundary conditions are of either isotropic or anisotropic interface type. This is a typical feature of superconductors of thickness smaller or equal to $\xi$. Nevertheless, looking at each particular choice of interfaces, we see that for parallelepiped covered by at least one thin layer of a superconductor with higher $T_{C}$ the demagnetization effect is stronger. For case 1, this effect could never be detected by using the 2D TDGL equations, since we do not have top and bottom faces. Once again, we notice the relevance of the 3D approach for the TDGL equations.

\section{Conclusions}

In conclusion, we have shown that anisotropic surface conditions described by different values of the de Gennes parameter can produce either a reduction or an enhancement of the thermodynamics properties of a three dimensional small superconducting parallelepiped in the presence of an external applied magnetic field. Critical magnetic fields, both for nucleation and full expulsion of vortices, have been studied and we found their dependence with different interfaces. The dependence of the vortex configurations with anisotropic surfaces were also investigated. Our findings show that the vortex configurations and the size of the vortices can be strongly influenced by the anisotropic surface conditions. For instance, we show that, for a sample of the same size, different boundary conditions can produce distinct giant vortex states (different vorticity and core size). This could not be entirely conclusive by using a 2D TDGL model, since in this case we would not have the top and bottom surfaces of the superconducting film.

\section{Acknowledgements}

ES thanks the Brazil Agency FAPESP for financial support (grant 2012/04388-00).

\section{References}

[1] H. Kupfer, G. Linker, G. Ravikumar, T. Wolf, A. Will, A.A. Zhukov, R. MeierHirmer, B. Obst, H. Wuhl, Phys. Rev. B 67 (2003) 064507.

[2] E.T. Filby, A.A. Zhukov, P.A.J. de Groot, M.A. Ghanem, P.N. Bartlett, V.V. Metlushko, Appl. Phys. Lett. 89 (2006) 092503.

[3] Z.L. Xiao, C. Han, W.K. Kwok, H.H. Wang, U. Welp, J. Wang, G. Crabtree, J. Am. Chem. Soc. 126 (2004) 2316

[4] I. Lukyanchuk, V.M. Vinokur, A. Rydh, R. Xie, M.V. Milošević, U. Welp, M. Zach, Z.L. Xiao, G.W. Crabtree, S.J. Bending, F.M. Peeters, W.K. Kwok, Nat. Phys. 11 (2015) 21
[5] A. Müller, M.V. Milošević, S.E.C. Dale, M.A. Engbarth, S.J. Bending, Phys. Rev. Lett. 109 (2012) 197003.

[6] M.A. Engbarth, S.J. Bending, M.V. Milošević, Phys. Rev. B 83 (2011) 224504.

[7] D. Glotov, Z. Angew. Math. Phys. 62 (2011) 891.

[8] Q. Du, M.D. Gunzburger, Physica D 69 (3-4) (1993) 215.

[9] S.J. Chapman, Q. Du, M.D. Gunzburger, Z. Angew. Math. Phys. 47 (3) (1996) 410.

[10] A.K. Elmurodov, D.Y. Vodolazov, F.M. Peeters, Europhys. Lett. 74 (2006) 151

[11] B. Xu, M. Milošević, F.M. Peeters, Phys. Rev. B 77 (2008) 144509.

[12] M. Doria, A.R. Romaguera, F.M. Peeters, Phys. Rev. B 75 (2007) 064505.

[13] Q. Du, J. Math. Phys. 46 (2005) 095109.

[14] Y. Chen, M. Doria, F.M. Peeters, Phys. Rev. B 77 (2008) 054511.

[15] J. Barba-Ortega, J.D. Gonzalez, Edson Sardella, J. Low Temp. Phys. 174 (96) (2014).

[16] J. Barba-Ortega, E. Sardella, J.A. Aguiar, Supercond. Sci. Technol. 24 (2011) 015001.

[17] F. Rogeri, R. Zadorosny, P.N. Lisboa-Filho, E. Sardella, W.A. Ortiz, Supercond. Sci. Technol. 26 (2013) 075005

[18] E.H. Brandt, Phys. Rev. B 72 (2005) 024529; J.R. Clem, E.H. Brandt, Phys. Rev. B 72 (2005) 174511.

[19] J. Barba-Ortega, E. Sardella, J.A. Aguiar, Phys. Lett. A 379 (2015) 732.

[20] W.V. Pogosov, Phys. Rev. B 65 (2002) 224511.

[21] W.D. Gropp, H.G. Kaper, G.K. Leaf, D.M. Levine, M. Palumbo, V.M. Vinokur, J. Comput. Phys. 123 (1996) 254.

[22] G. Buscaglia, C. Bolech, C. Lopez, in: J. Berger, J. Rubinstein (Eds.), Connectivity and Superconductivity, Springer, Heidelberg, 2000.

[23] M.V. Milošević, R. Geurts, Physica C 470 (2010) 791.

[24] P.G. de Gennes, Superconductivity of Metals and Alloys, Addison-Wesley, New York, 1994.

[25] P.G. de Gennes, J. Matricon, Rev. Mod. Phys. 36 (1964) 45.

[26] E.A. Andrushin, V.L. Ginzburg, A.P. Silin, Usp. Fiz. Nauk 163 (1997) 105.

[27] R.O. Zaitsev, Zh. Eksp. Teor. Fiz. 48 (1965) 1759.

[28] J. Simonin, Phys. Rev. B 33 (1986) 7830.

[29] H.J. Fink, S.B. Haley, C.V. Giuraniuc, V.F. Kozhevnikov, J.O. Indekeu, Mol. Phys. 103 (2005) 21.

[30] V.V. Moshchalkov, X.G. Qiu, V. Bruyndoncx, Phys. Rev. B 55 (1997) 11793

[31] V.A. Schweigert, F.M. Peeters, P.S. Deo, Phys. Rev. Lett. 81 (1998) 2783.

[32] V.A. Schweigert, F.M. Peeters, Phys. Rev. B 57 (1998) 13817.

[33] V. Bruyndoncx, L. Van Look, M. Verschuere, V.V. Moshchalkov, Phys. Rev. B 60 (1999) 10468;

A.K. Geim, S.V. Dubonos, I.V. Grigorieva, K.S. Novoselov, F.M. Peeters, V.A. Schweigert, Nature (London) 407 (2000) 55.

[34] J.J. Palacios, Phys. Rev. Lett. 84 (2000) 1796.

[35] A.K. Geim, S.V. Dubonos, J.J. Palacios, Phys. Rev. Lett. 85 (2000) 1528.

[36] S.V. Yampolskii, F.M. Peeters, Phys. Rev. B 62 (2000) 9663; E. Montevecchi, J.O. Indekeu, Phys. Rev. B 62 (2000) 14359

[37] B.J. Baelus, F.M. Peeters, Phys. Rev. B 65 (2002) 104515; E. Sardella, A.L. Malvezzi, P.N. Lisboa-Filho, W.A. Ortiz, Phys. Rev. B 74 (2006) 014512.

[38] T. Nishio, Q. Chen, W. Gillijns, K. De Keyser, K. Vervaeke, V.V. Moshchalkov, Phys. Rev. B 77 (2008) 012502;

I.V. Grigorieva, W. Escoffier, V.R. Misko, B.J. Baelus, F.M. Peeters, L.Y. Vinnikov, S.V. Dubonos, Phys. Rev. Lett. 99 (2007) 147003.

[39] T. Cren, L. Serrier-Garcia, F. Debontridder, D. Roditchev, Phys. Rev. Lett. 107 (2011) 097202;

A. Kanda, B.J. Baelus, F.M. Peeters, K. Kadowaki, Y. Ootuka, Phys. Rev. Lett. 93 (2004) 257002;

Ben Xu, M.V. Milošević, Shi-Hsin Lin, F.M. Peeters, B. Jankó, Phys. Rev. Lett. 107 (2011) 057002 